\title{
FeLoBAL quasars: evolution, orientation or just oddBALs?
}

\author{
Daniel Lawther ${ }^{1}$, Marianne Vestergaard ${ }^{1,2}$ and Xiaohui Fan ${ }^{2}$ \\ ${ }^{1}$ Dark Cosmology Centre, Niels Bohr Institutet, Denmark \\ email: danielpe@dark-cosmology.dk \\ ${ }^{2}$ Steward Observatory, University of Arizona, USA
}

\begin{abstract}
FeLoBAL quasars may be an early evolutionary stage of merger-triggered quasar activity. We test this hypothesis using HST imaging of four FeLoBAL quasars. The host galaxy colors are consistent with early-type quiescent galaxies or heavily-obscured starbursts.
\end{abstract}

Keywords. galaxies: active, quasars: general, quasars: absorption lines

\section{Introduction}

Broad absorption line (BAL) quasars display strong, high-velocity absorption lines in their UV/optical spectra. Iron low-ionization broad absorption line quasars (FeLoBALs) are a rare subtype that also show absorption due to to excited Fe II and Fe III transitions.

The origin of the strong absorption in FeLoBAL objects is unknown. In the context of an orientation-based unification scenario (e.g., Urry and Padovani 1995), BAL quasars are objects for which our line of sight intersects a high-velocity outflow - perhaps a wind launched from the disk (e.g., Elvis 2012). An alternative scenario suggested by Voit et al.(1993) is that FeLoBALs represent young (perhaps merger-triggered) quasars observed in the process of breaking through a cocoon of enshrouding dust and gas.

\section{HST imaging observations}

To test this issue, we observed four of the most heavily absorbed 'overlapping-trough' FeLoBAL quasars (Hall et al.2002) at $z \approx 1.5$ in rest-frame ultraviolet and optical bands using the Hubble Space Telescope. We provide basic information on the HST observations in Table 1. We selected UV photometric filters that sample the BAL absorbing troughs, so as to minimize the contribution of the active nucleus to the total light, aiding host galaxy detection. Our aim was to determine the nature of the FeLoBAL host galaxies: are they quiescent ellipticals, as seen for bright quasars in the local Universe, or galaxies showing signs of recent merger activity, as the Voit et al.(1993) scenario suggests?

\section{Modeling nuclear and host galaxy contributions}

We use GALFIT (Peng 2010) to model the nuclear and extended emission in each bandpass. We determine the uncertainties on the measured nuclear and host galaxy magnitudes using a sample of simulated quasar observations generated using FERENGI (Barden et al.2008). In the rest-frame UV we obtain upper limits on the host magnitudes. In the rest-frame optical we detect a compact, bright host galaxy for the FeLoBAL J1154+0300. We marginally detect faint host galaxies for two other quasars, and obtain an upper limit for the last object. Our data and results will be presented in a forthcoming paper (Lawther et al.2015, in prep.). 
Table 1. HST imaging data

\begin{tabular}{lccccccc}
\hline $\begin{array}{l}\text { Object } \\
\text { name }\end{array}$ & $\mathbf{z}$ & $\begin{array}{c}\text { ACS/WFC } \\
\text { filter }\end{array}$ & $\begin{array}{c}\text { ACS (UV) } \\
\text { magnitude }\end{array}$ & $\begin{array}{c}\text { NICMOS } \\
\text { filter }\end{array}$ & $\begin{array}{c}\text { NICMOS (Opt) } \\
\text { magnitude }\end{array}$ & $\begin{array}{c}\text { Orbits } \\
\text { (ACS,NIC) }\end{array}$ & $\begin{array}{c}\text { Exp. time [s] } \\
\text { (ACS,NIC) }\end{array}$ \\
\hline J0300+0048 & 0.89 & F $435 \mathrm{~W}$ & 20.0 & F110W & 16.4 & 1,1 & 2185,2560 \\
J1154+0300 & 1.46 & F550M & 21.1 & F110W & 17.3 & 2,2 & 5141,5367 \\
J0819+4209 & 1.93 & F625W & 22.7 & F160W & 19.2 & 3,3 & 8150,8695 \\
J1730+5850 & 2.04 & F625W & 21.9 & F160W & 18.0 & 4,4 & 6517,8191 \\
\hline
\end{tabular}
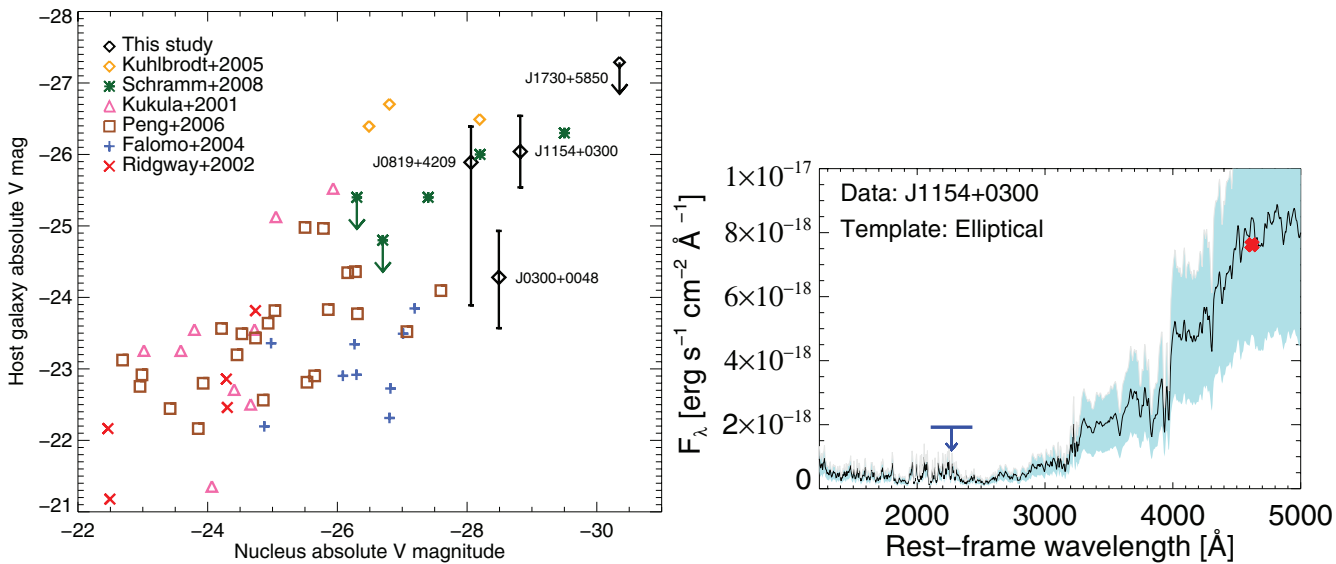

Figure 1. Left: Comparison of our sample with literature studies in the V band nuclear luminosity versus host galaxy luminosity plane. Our objects are shown as black diamonds; the uncertainties on our host galaxy magnitudes are based on simulations. Right: The flux density of the host galaxy of FeLoBAL J1154+0300 in the rest-frame optical (red) and an upper limit on the host galaxy flux in the UV (blue arrow). Also shown is a template spectrum of an elliptical galaxy, scaled to our optical flux density. The blue shaded area shows the uncertainty on the template scaling due to our measurement uncertainties, as determined using simulated observations.

\section{Results}

The V-band luminosities of the host galaxies of our FeLoBAL quasars are consistent with those of 'normal', non-BAL quasars. Our constraints on the UV-optical color are consistent with early-type host galaxies (Figure 1, right panel). However, we are unable to exclude the possibility of a strongly dust-reddened starburst galaxy. We are in the process of obtaining additional data to further investigate this issue.

\section{References}

Barden, M., Jahnke, K. and Häussler, B. 2008, ApJS, 175, 105B

Elvis, M. 2012, $A S P C$, v.460, p.186E

Hall, P.B. et al., 2002, ApJS, 141, 267H

Peng et al. 2010, AJ, 139, 2097

Urry, C.M. and Padovani, P. 1995, PASP, v.107, p.803

Voit, G. M. and Weymann, R. J. and Korista, K. T. 1993, ApJ, 413, 95V 